\title{
Near-Infrared Wavefront Sensing
}

\author{
Peter Wizinowich ${ }^{1, *}$, Mark Chun $^{2}$, Dimitri Mawet ${ }^{3}$, Guido Agapito ${ }^{4}$, Richard Dekany ${ }^{3}$, Simone \\ Esposito $^{4}$, Thierry Fusco ${ }^{5,6}$, Olivier Guyon ${ }^{7}$, Donald Hall ${ }^{2}$, Cedric Plantet ${ }^{5,8}$, Francois Rigaut ${ }^{9}$ \\ 1. W. M. Keck Observatory, 65-1120 Mamalahoa Hwy., Kamuela, HI, USA 96743 \\ 2. Institute for Astronomy, University of Hawaii, 640 N. A'ohoku Place, Hilo, HI, USA 96720 \\ 3. Astronomy Dept., Caltech, 1200 E. California Blvd., Pasadena, CA, USA 91125 \\ 4. Arcetri Astrophysical Observatory, Largo E. Fermi 5, 50125-Firenze, Italy
}

5. Aix Marseille Univ., CNRS, Laboratoire d'Astrophysique de Marseille UMR 7326, 13388 Marseille, France

6. Onera, the French Aerospace Lab, 92322 Chatillon, France

7. Subaru Telescope, National Astronomical Observatory of Japan, 650 N, A'ohoku Place, Hilo, HI, USA 96720

8. Universite Grenoble Alpes, CNRS, IPAG, F-38000, Grenoble, France

9. Research School of Astronomy \& Astrophysics, Australian National University, Canberra, Australia

\begin{abstract}
We discuss the advantages of wavefront sensing at near-infrared (IR) wavelengths with low-noise detector technologies that have recently become available. In this paper, we consider low order sensing with laser guide star (LGS) adaptive optics (AO) and high order sensing with natural guide star (NGS) AO. We then turn to the application of near-IR sensing with the W. M. Keck Observatory (WMKO) AO systems for science and as a demonstrator for similar systems on extremely large telescopes (ELTs). These demonstrations are based upon an LGS AO near-IR tip-tilt-focus sensor and our collaboration to implement a near-IR pyramid wavefront sensor (PWFS) for a NGS AO L-band coronagraphic imaging survey to identify exoplanet candidates.
\end{abstract}

Keywords: adaptive optics, wavefront sensing, tip-tilt sensing, near infrared, pyramid, Keck, LIFT

\section{INTRODUCTION}

Near-infrared wavefront sensing is a critical technology for science with AO on current and future telescopes. It enables high contrast science of exoplanets and dust obscured regions, and high sky coverage for extragalactic science. It can be used to extend the performance of NGS AO to fainter (redder) targets and to increase the sky coverage of laser guide star (LGS) AO. Furthermore, it allows the application of optimal wavefront sensing approaches (e.g. pyramid and Zernike wavefront sensing) due to the AO correction at near-IR wavelengths. All of the extremely large telescopes (ELTs) are planning to use near-IR wavefront sensing as part of their AO facilities.

Infrared cameras have been used as phase and angle sensors on astronomical interferometers for many years (e.g. [1]). The VLT NACO Shack-Hartmann camera is the only high-order AO near-IR wavefront sensor in operational use [2] and a near-IR PWFS was demonstrated on-sky with the Calar Alto AO system [3]. The limited use of near-IR wavefront sensing has been due to the high read noise, and high cost, of these detectors which limited them to bright targets. The high read noise limitation has recently been removed with the introduction of new approaches and technologies.

In terms of new approaches to reduce read noise, a near-IR tip-tilt sensor, based on multiple reads of small windows on a Teledyne H2RG detector, has been implemented with the Keck I LGS AO system [4]; however there is still a high procurement cost associated with this detector. In terms of new technologies for low read noise and lower cost, both CEA-Leti/Sofradir and Selex have demonstrated the production of electron initiated avalanche photodiode arrays (APDs) [5]. A CEA-Leti/Sofradir RAPID detector is in operational use as part of the VLTI PIONIER instrument [6]. A Selex SAPHIRA array has been demonstrated for lucky imaging on the IRTF [7] and for tip and tilt with the Robo-AO system [8], and for wavefront sensing and fringe tracking on GRAVITY [9].

In this paper we report on the status of lab, on-sky and analytical results for near-IR sensors used for tip-tilt and low order modes for LGS AO, and high order pyramid wavefront sensing for NGS AO (section 2). We also report on the conceptual design of a combined near-IR tip-tilt and PWFS sensor for the Keck II AO system (section 3).

Adaptive Optics Systems V, edited by Enrico Marchetti, Laird M. Close, Jean-Pierre Véran, Proc. of SPIE Vol. 9909, 990915 (C) 2016 SPIE · CCC code: 0277-786X/16/\$18 · doi: 10.1117/12.2233035 


\section{ANALYSIS AND RESULTS}

\subsection{Selex Detector Performance}

The initial Selex 320 x 256 at $24 \mu$ m pitch e-APD SAPHIRA arrays utilized Liquid Phase Epitaxy (LPE) HgCdTe. A development program initiated by ESO and carried on by UH switched to Metal Organic Vapor Phase Epitaxy (MOVPE) HgCdTe. This technology, which enables full band gap engineering of the APD, has allowed dramatic improvements in the quality and performance of the SAPHIRA arrays [10]. The most recent models have achieved superb cosmetic quality at avalanche gains as high as 600 (at 20 volts bias!). These arrays have 32 parallel video outputs organized as 32 adjacent pixels in a row and, for windowing, the readout allows addressing of any modulo 32 set in the array. The readout is inherently capable of pixel rates well in excess of $10 \mathrm{MHz}$ although the highest rate achieved to date are $5 \mathrm{MHz}$ with the ESO controller. This corresponds to a full frame rate of $2 \mathrm{kHz}$ and $10 \mathrm{KHz}$ for a 128 x 128 subarray with deep sub-electron read noise. The quantum efficiency is $70 \%$ and the mean read noise at 2000 frames per second and a gain of 60 is $<1 \mathrm{e}$.

The technology is readily scalable to larger format. Selex and its partners are actively working towards $1 / 4$ to one megapixel class formats [11].

\subsection{Tip-tilt Sensing for LGS AO}

The performance of the Keck I near-IR tip-tilt sensor with LGS AO has been measured [12] and some sample measurements are shown in Table 1. The near-IR tip-tilt sensor manages to maintain better tip-tilt performance than the visible tip-tilt sensor as the NGS gets fainter. Both sensors can use stars up to $\sim 50$ " off-axis. The near-IR sensor uses a $2048 \times 2048$ pixel detector and off-axis NGS are acquired by reading out the appropriate small (e.g. 4x4 pixel) region on the detector; while the visible sensor must be moved off-axis to acquire the NGS. A potential advantage of this near-IR sensor is that multiple NGS can be used by reading out multiple regions in order to reduce tip-tilt anisoplanatism (this mode is implemented but needs on-sky testing).

Table 1: Keck LGS AO H-band performance with the visible and near-IR tip-tilt sensors for three different NGS. For these measurements the LGS was pointed at the tip-tilt NGS, while the performance was measured on a nearby star.

\begin{tabular}{|c|c|c|c|c|c|c|}
\hline \multirow{2}{*}{$\begin{array}{l}\text { Tip-tilt } \\
\text { Sensor }\end{array}$} & \multicolumn{2}{|c|}{$\begin{array}{c}R=12.0 \& \mathrm{~K}=11.1 \\
8.6 " \text { off-axis }\end{array}$} & \multicolumn{2}{|c|}{$\begin{array}{c}R=13.0 \& \mathrm{~K}=11.5 \\
6.4^{\prime \prime} \text { off-axis }\end{array}$} & \multicolumn{2}{|c|}{$\begin{array}{c}\mathrm{R}=15.5 \& \mathrm{~K}=13.8 \\
\text { on-axis }\end{array}$} \\
\hline & Strehl & FWHM (mas) & Strehl & FWHM (mas) & Strehl & FWHM (mas) \\
\hline Visible & 0.32 & 45 & 0.22 & 48 & 0.20 & 56 \\
\hline Near-IR $4 \times 4$ & 0.35 & 41 & 0.29 & 40 & 0.36 & 43 \\
\hline
\end{tabular}
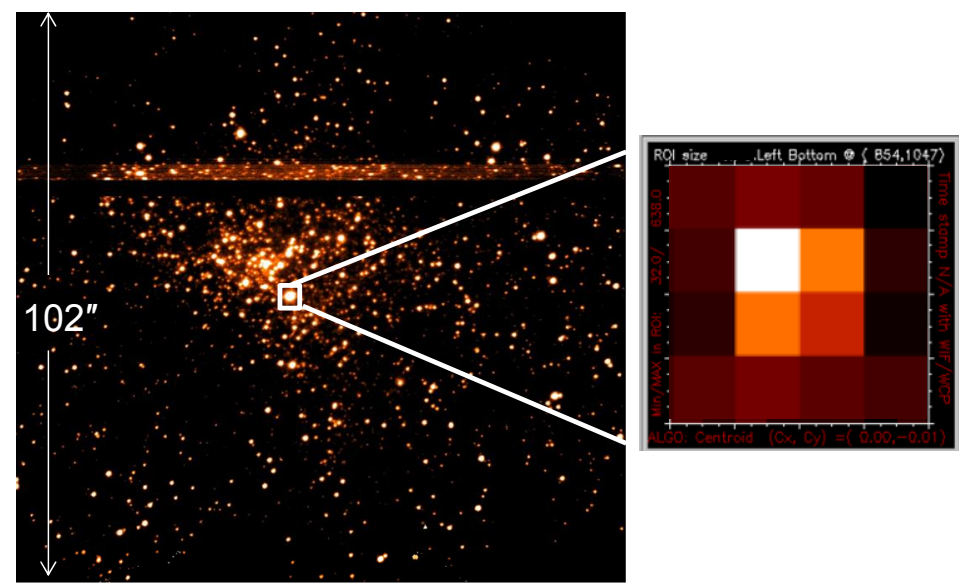

Figure 1: Left: A Ks-band image of the Galactic center taken with the Keck I near-IR tip-tilt sensor. Right: A 4x4 pixel (0.1"x0.1") region of interest anywhere on the detector can be read out non-destructively multiple times to reduce the measurement error on the tip-tilt measurement.

In order to determine the relative merits of the H2RG and e-APDs we can compare their signal-to-noise ratios (SNR). 
For the e-APDs the

$$
S N R=\frac{\eta \mathrm{S}}{\sqrt{F\left[\eta\left(\mathrm{S}+\mathrm{S}_{B}\right)+\mathrm{S}_{D}\right]+\mathrm{n}\left(\frac{\sigma_{R}}{\mathrm{G}}\right)^{2}}}
$$

where $\eta$ is the detector quantum efficiency; $S, S_{B}$ and $S_{D}$ are the signal due to the star, background (sky and thermal) and dark current on the pixels used for the measurement; $\mathrm{F}$ is the excess noise caused by the amplification; $\mathrm{n}$ is the number of pixels; $\sigma_{R}$ is the read-noise per pixel; and $\mathrm{G}$ is the e-APD gain. Note that the calculation of the stellar signal in the $\mathrm{n}$ pixels includes a multiplication by the Strehl ratio. The effect of the e-APD gain is to effectively reduce the read-noise at the expense of some excess noise in the detected photons.

A plot of H-band SNR versus magnitude is shown in Figure 2. The e-APD outperforms the H2RG for the 4x4 pixel case. The higher SNR performance for the e-APD system allows an e-APD system to be operated at higher bandwidth than an H2RG system while maintaining the H2RG SNR level. The APD's performance advantage over the H2RG increases with the number of pixels (e.g. the APD's can be used for a high order wavefront sensor). There is little performance difference between the two detectors in the $2 \times 2$ pixel case.

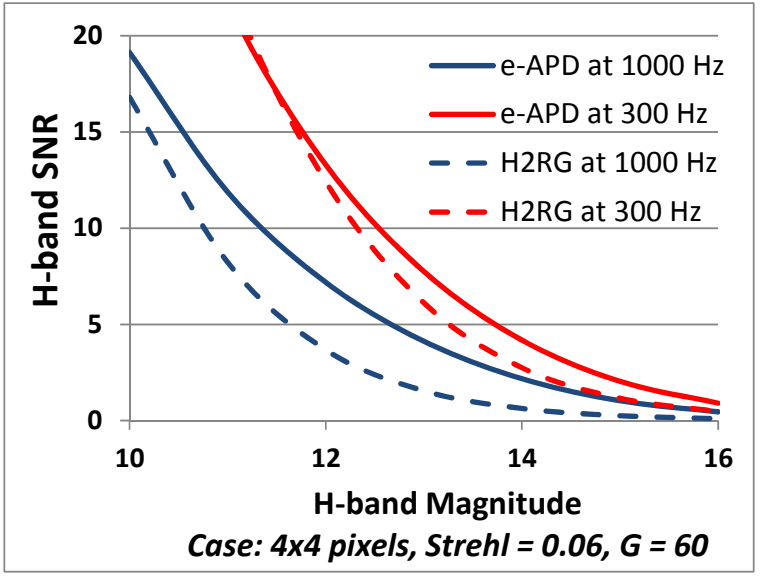

Figure 2: H-band SNR versus magnitude. Additional assumptions: $49 \%$ throughput and $Q=70 \%$ for Selex, $45 \%$ throughput and $Q=$ and $85 \%$ for $H 2 R G$, and $F=1.2$.

\subsection{Low Order Focal Plane Wavefront Sensing for LGS AO}

The LGS wavefront sensor measurements include focus changes due to variations in the altitude of the sodium layer and subaperture tilt changes due to variations in the structure of the sodium layer and the telescope pupil orientation. The Keck LGS AO system [13] uses a visible low bandwidth wavefront sensor (LBWFS) to measure a NGS (i.e. truth) and hence provide focus and centroid corrections to the LGS wavefront sensor. The LBWFS can limit LGS AO performance and observing efficiency especially on faint NGS where 1 to 2 minute exposures can be required.

The errors measured by the LBWFS are low order aberrations (e.g. astigmatism, coma and spherical). Focal plane wavefront sensing in the near-IR, where the image is partially AO-corrected, offers a potentially more efficient alternative to the LBWFS, especially if this could be done with the data from the near-IR tip-tilt sensor.

The focal plane wavefront sensor algorithm LIFT [14] uses a single astigmatic image to estimate tip/tilt, focus and other low order aberrations. LIFT has been tested with calibration sources on the Keck I near-IR tip-tilt sensor and the Keck II science camera NIRC2 [15]. The near-IR tip-tilt sensor's 50 mas pixels are under-sampled ( 0.45 Nyquist sampling at K), while the NIRC2 10 mas pixels provide well sampled images. The AO loop was closed on a calibration source and Zernike aberrations modes, in addition to the astigmatism offset needed by LIFT, were inserted by changing the reference slopes on the wavefront sensor. Tip-tilt was successfully retrieved on both instruments (Figure 3a and Figure 4a). Some non-linearities were observed on TRICK for the estimation of focus and $0^{\circ}$ astigmatism (Figure $3 \mathrm{~b}$ and d), but most amplitudes are well estimated. The $45^{\circ}$ astigmatism is well estimated in positive values (Figure $3 \mathrm{c}$ ). The inflection point is expected and corresponds to the opposite of the inserted astigmatism offset. These results can be improved with better knowledge of the imaging model, which is fundamental at such a sampling. 

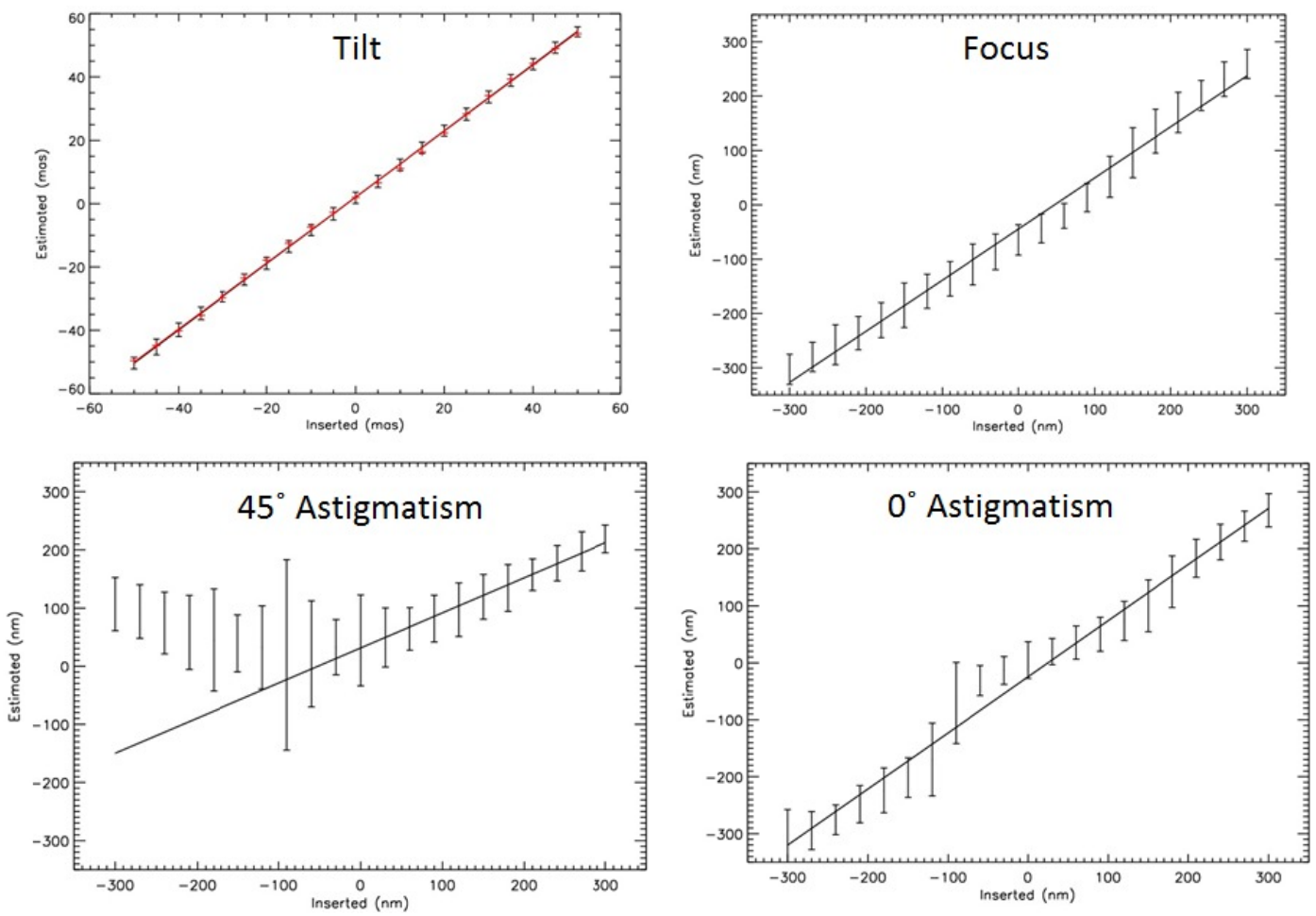

Figure 3: Estimation of ramps of pure Zernike modes by LIFT on TRICK. Sampling: 0.45 Nyquist sampling. The blue plot in (a) is a center of gravity estimation.
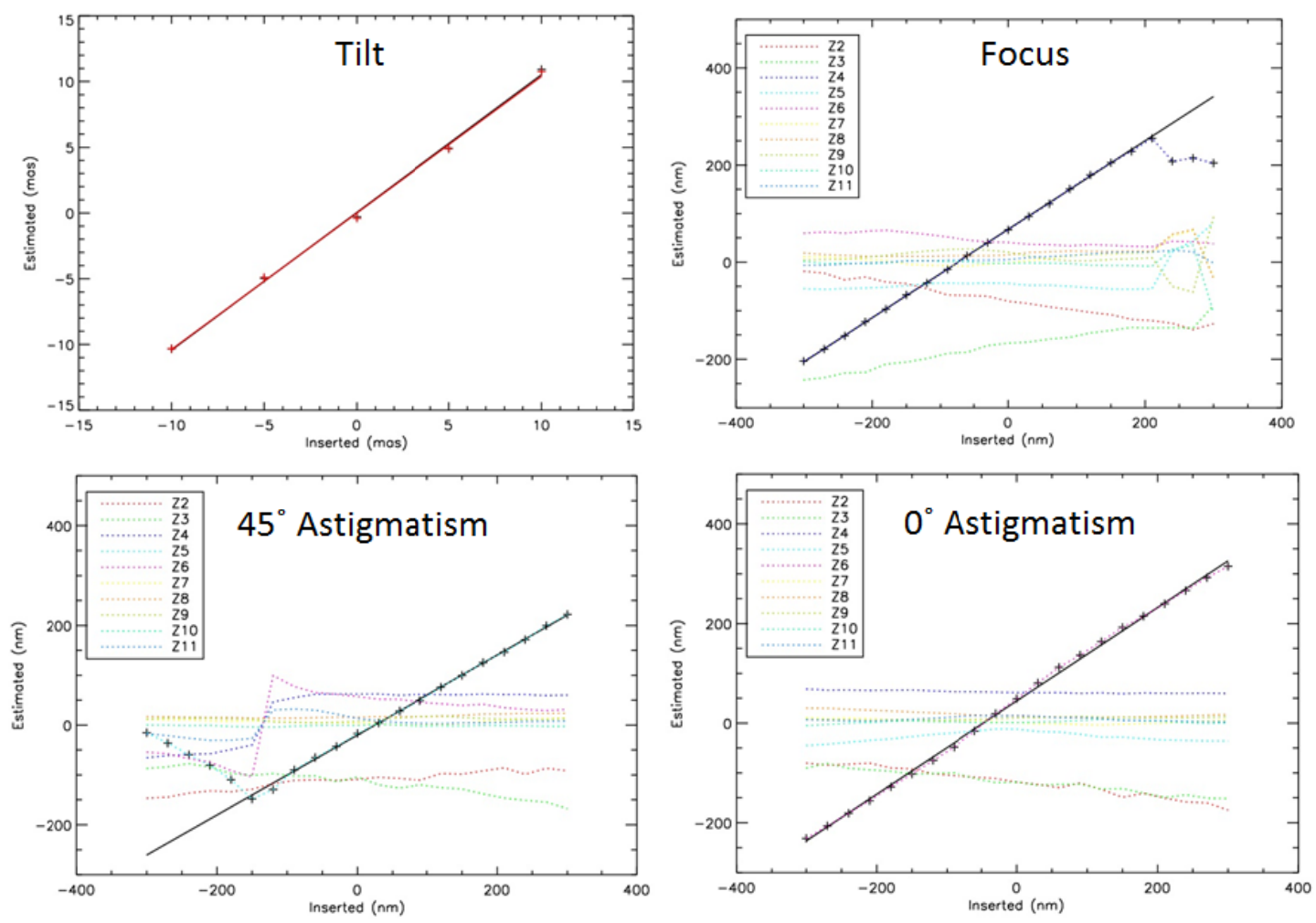

Figure 4: Estimation of ramps of pure Zernike modes by LIFT with NIRC2. Sampling: 1.96 Nyquist sampling. The blue plot in (a) is a center of gravity estimation. 
The good NIRC2 sampling allowed the estimation of low orders up to 10 modes. The focus and both astigmatisms were well retrieved within the linearity range (Figure 4), with the other modes being constant and close to zero, except for tiptilt. The coupling with tip-tilt is due to a misalignment of the pupil with the deformable mirror (which does not have an impact on sky, and thus has not been corrected). Also, a scaling factor of $\sim 0.8$ to 0.9 has to be applied to get the correct amplitude. This may also be due to the pupil misalignment, but other error sources, such as an incorrect projection of Zernike modes on the deformable mirror, are being investigated. These tests have also been performed with different pupil shapes (using masks in NIRC2 including the Keck primary mask) and at different wavelengths, with similar results. LIFT appears to be robust to errors linked to complex imaging models (e.g. very low sampling, pupil misalignment and a complex pupil shape) present in the Keck's AO systems.

An analysis of tip-tilt and focus errors versus star magnitude has been performed to understand the optimal pixel size for a new near-IR tip-tilt sensor for Keck II. The analysis used a similar approach to that of [14] described in the next section. The results for a $300 \mathrm{~Hz}$ sample rate are shown in Figure 5; in practice the focus measurements would be integrated to average out seeing before being applied as corrections. For bright NGS aliasing dominates the tip-tilt and focus errors while detector noise dominates for faint NGS. The aliasing is a function of Strehl ratio.

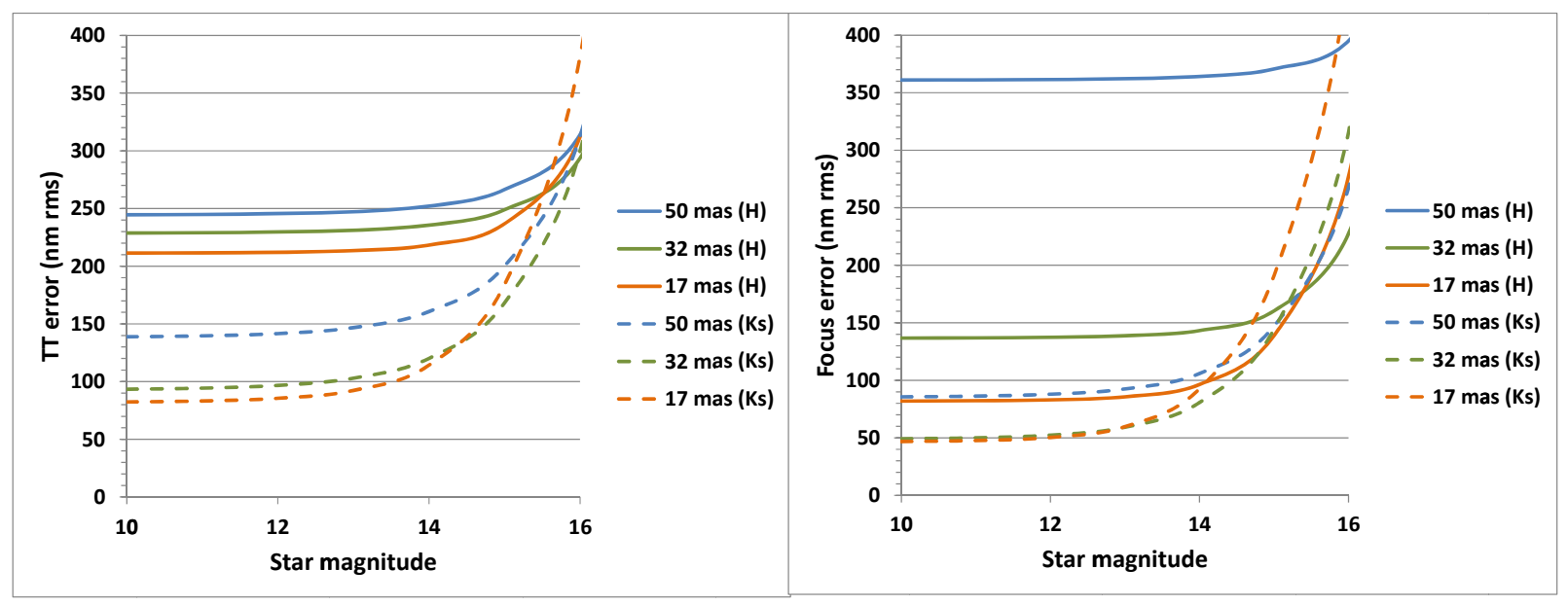

Figure 5: Wavefront error as a function of pixel size (mas) and magnitude, in $\mathrm{H}$ or Ks-band, at $300 \mathrm{~Hz}$ sampling assuming an A0 star and Ks-band Strehl ratio of 0.25. Left: tip-tilt error. Right: focus error.

As shown in Figure 6, LIFT can also provide a significant tip-tilt performance improvement for faint stars, versus the simple centroiding algorithm used with the Keck I near-IR tip-tilt sensor.

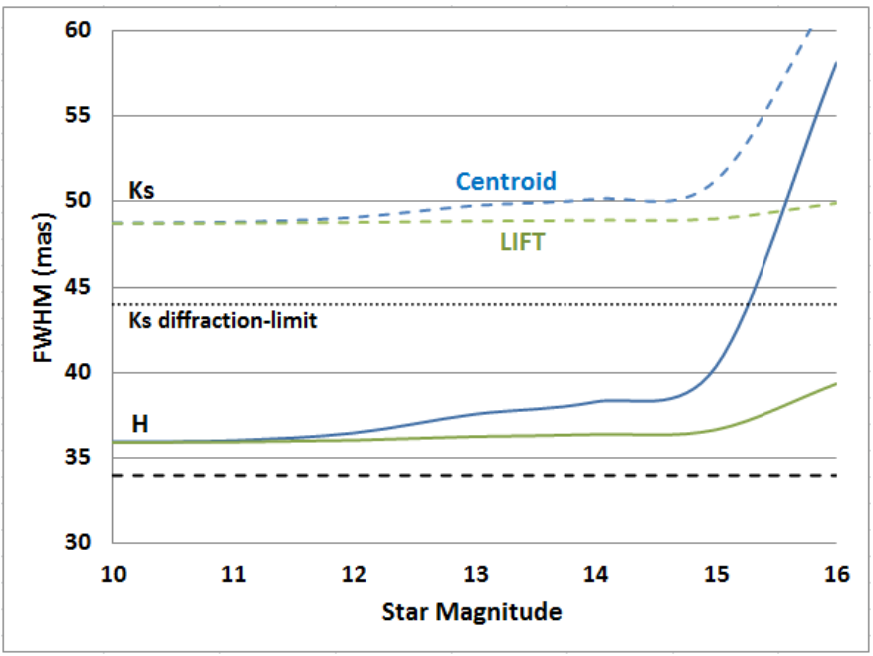

Figure 6: NGS FWHM on the science camera for a centroid algorithm and for LIFT. The Ks-band science images are obtained with the Keck I near-IR tip-tilt sensor operating in $\mathbf{H}$-band, while the $\mathrm{H}$-band science images are obtained with Ks-band tip-tilt sensing. The sampling rate was reduced from $800 \mathrm{~Hz}$ at $10^{\text {th }}$ magnitude to $100 \mathrm{~Hz}$ at $15^{\text {th }}$ and $16^{\text {th }}$ magnitude. The Ks-band Strehl ratio was 0.25 and an $\mathrm{A0}$ star was assumed. 


\subsection{Pyramid Wavefront Sensing for NGS AO}

Our case for a near-IR pyramid wavefront sensor (PWFS) is driven by the desire to study planet formation around obscured and red objects, such as young stars in distant star forming regions, and late-type stars, including $\mathrm{M}$ dwarfs. The abundance of M-type stars, their low close binary fraction, and the ubiquitous presence of massive proto-planetary disks at young ages imply that they are common sites of planet formation. Unfortunately the faintness of these stars at optical wavelengths has made them difficult to observe at the required contrast and spatial resolution to detect exoplanets with current AO systems. These stars are however sufficiently bright in the near-IR to be used as AO guide stars. The planet to star contrast ratio is particularly favorable in L-band and a suitable L-band vortex coronagraph has recently been implemented with NIRC2 and the Keck II AO system [16]. The limiting magnitude gain by going to near-IR wavefront sensing and the resultant probability of planet detection with the L-band vortex coronagraph is shown in Figure 7.

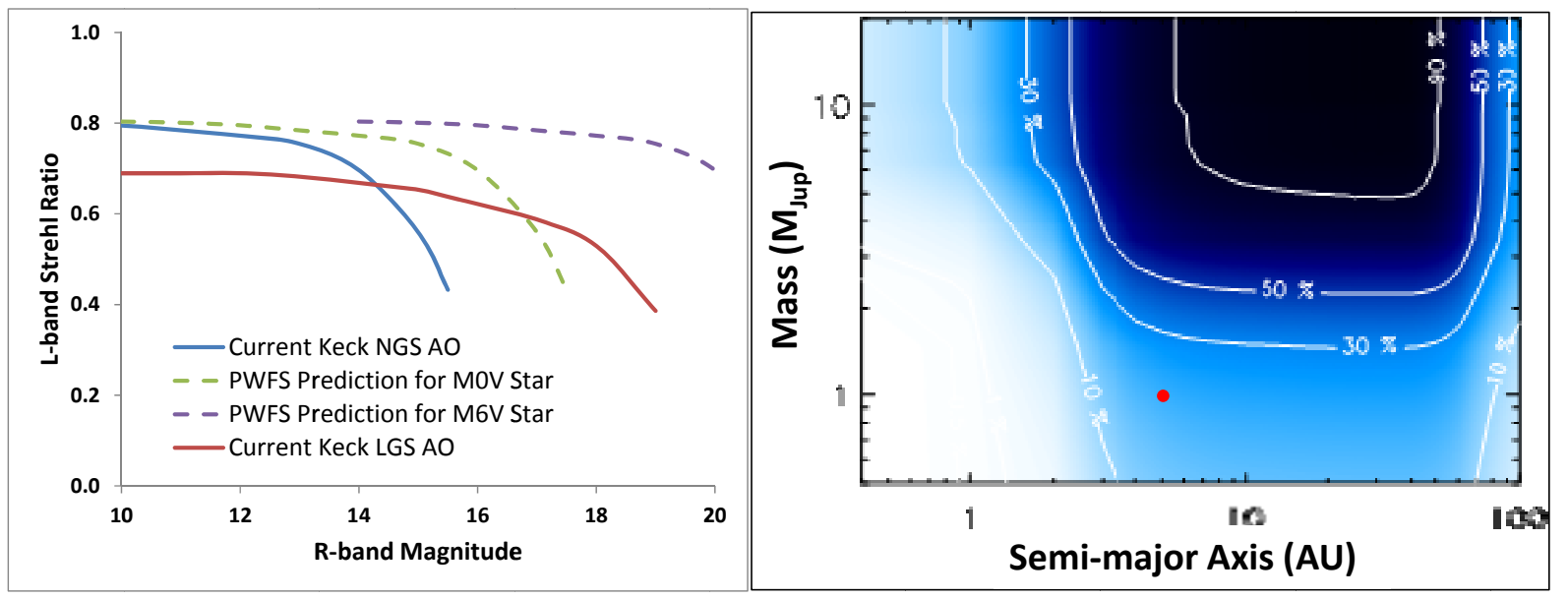

Figure 7: Current and predicted L-band Strehl ratios versus the R-magnitude of an on-axis guide star under median conditions. The current Keck NGS AO system uses a visible Shack-Hartmann wavefront sensor. The predicted NGS AO performance is for an efficient low-noise infrared PWFS using MOV and M6V spectral types guide stars, respectively. Right: Survey completeness, assuming the AO performance shown on the left and the performance model for the Keck L-band vortex coronagraph. The red dot is where Jupiter would sit in this plot.
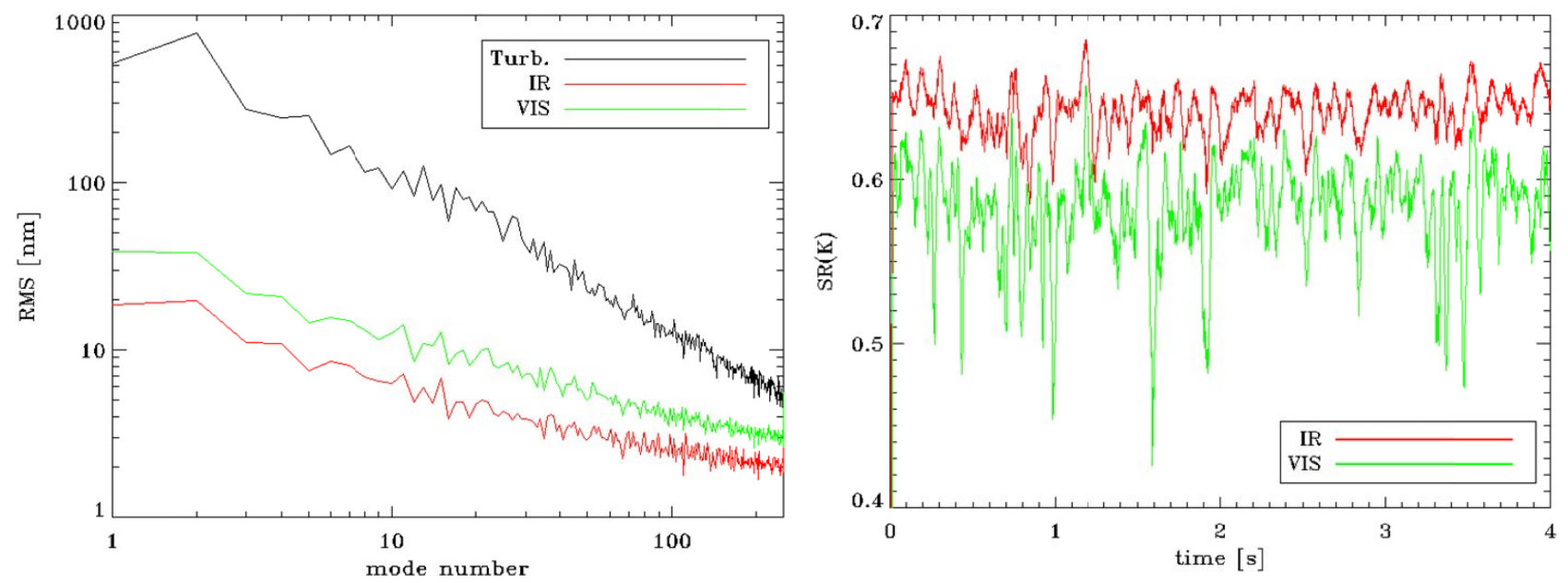

Figure 8: Simulated Keck AO performance versus PWFS wavelength. The plot at left shows the rms wavefront error versus mode number for Mauna Kea turbulence (black curve) and the Keck AO-corrected residual using a near-IR (red curve) and visible (green curve) PWFS. The plot at right shows the K-band Strehl ratio for the nearIR and visible PWFS versus time. The read-noise of both the visible $(750 \mathrm{~nm})$ and near-IR $(1.6 \mu \mathrm{m})$ detectors was assumed to be 1e- and a bright NGS (400 photons/ms/subaperture) was assumed in both cases. The number of corrected modes (250) is limited by the existing Keck AO deformable mirror. 
Given the high performance of visible PWFS it is natural to consider a near-IR PWFS now that the e-APD arrays are available. Initial PWFS simulations have been performed to understand the relative merits of a near-IR PWFS versus a visible PWFS for the Keck AO system given Mauna Kea turbulence. The modal turbulence and AO-corrected residuals are plotted in Figure 8 along with the resultant K-band Strehl ratio versus time. The near-IR PWFS outperforms the visible PWFS even for this case which assumes the same signal and read-noise; the AO-corrected rms residual wavefront error is $237 \mathrm{~nm}$ with the near-IR sensor versus $263 \mathrm{~nm}$ with a visible sensor. The near-IR sensor's performance advantage will increase when using typical stars which have higher flux in the near-IR and when more realistic visible CCD read-noise is considered.

The predicted wavefront error, including measurement and bandwidth errors, is plotted in Figure 9, for a near-IR PWFS. A bandwidth error of $100 \mathrm{~nm}$ rms was assumed at $100 \mathrm{~Hz}$ and was scaled as $\mathrm{T}_{\mathrm{int}}^{5 / 6}$ versus bandwidth. The total predicted variance of the wavefront estimation error in $\mathrm{rad}^{2}$ is given by [14] as

$$
\sigma_{e s t}^{2}=\frac{\alpha F}{\eta n_{p h}}+\beta\left(\frac{\sigma_{d e t}}{\eta n_{p h}}\right)^{2},
$$

where $n_{p h}$ is the number of photons incident on the pyramid during an integration. For the near-IR PWFS, the noise propagation coefficients, $\alpha$ and $\beta$, were computed for a maximum likelihood estimation of 200 Karhunen-Loeve modes, assuming a diffraction-limited spot and using the approach of [17]. These coefficients increase with the radii of modulation of the spot on the pyramid. The calculated values for a modulation of $3 \lambda / \mathrm{D}$ are $\alpha=536$ and $\beta=753670$. For the SAPHIRA detector an excess noise factor of $F=1.25$ and a quantum efficiency of $\eta$ $=0.7$ were used. The equivalent detector noise is computed as

$$
\sigma_{\text {det }}=\frac{\sqrt{\sigma_{\text {ron }}^{2}+F G^{2} i_{\text {dark }} T_{\text {int }}+F G^{2} T_{i n t} \eta\left(F_{p i x, t h}+F_{p i x, s k y}\right)}}{G},
$$

where $\sigma_{\text {ron }}=24 \mathrm{e}$ - and $i_{\text {dark }}=504 \mathrm{e}-/ \mathrm{s}$ are the read-out noise and dark current for unit gain, $G=30$ is the avalanche gain and $T_{\text {int }}$ is the integration time. The Hband thermal and sky background flux were taken as $F_{\text {pix }, \text { th }}=15$ photons $/ \mathrm{s} /$ pixel and $F_{p i x, s k y}=261$ photons/s/pixel, respectively.

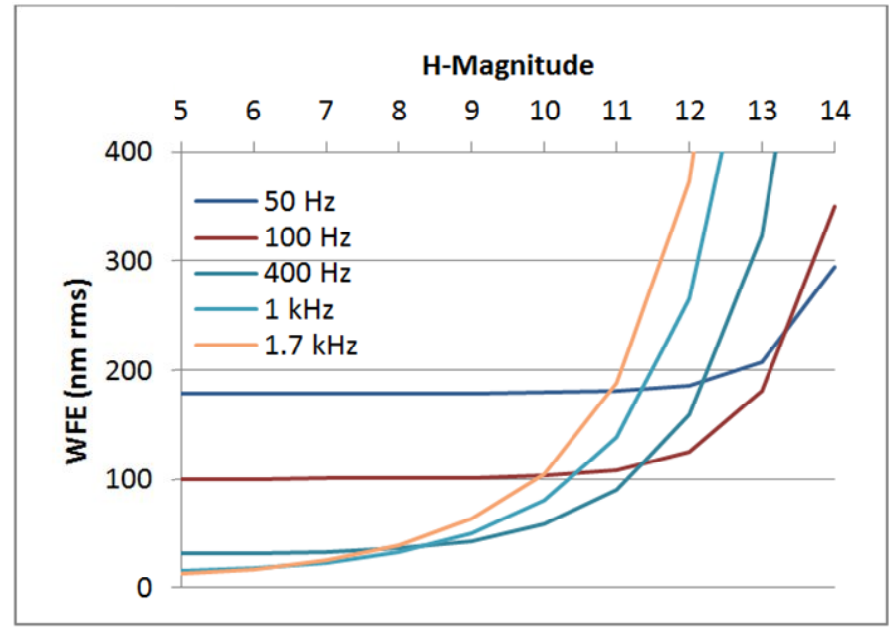

Figure 9: Combined measurement and bandwidth error

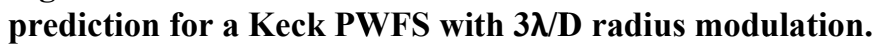

A demonstration of a near-IR (H-band) pyramid wavefront sensor based on a SAPHIRA detector and a UH camera was performed with the Subaru SCExAO system in October 2015. The sensor performed well in initial unmodulated lab testing (see Figure 10). However, because of the limited frame rate of the readout electronics the loop could only be run at $100 \mathrm{~Hz}$ which severely hampered the on-sky performance.

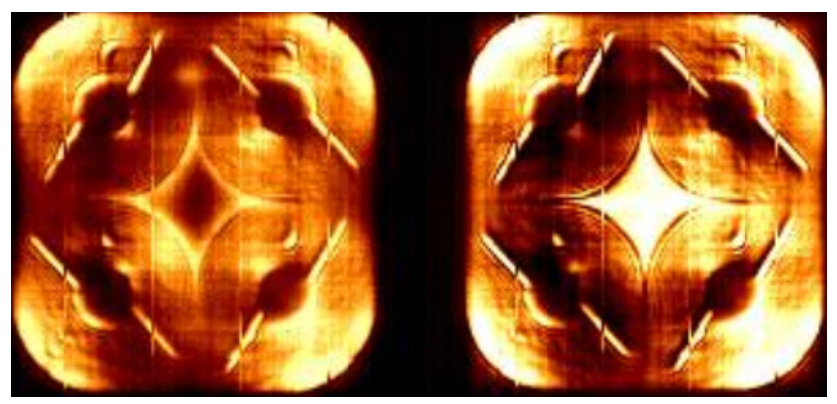

Figure 10: Near-IR pyramid wavefront sensor laboratory images of the four overlapping pupils from the Subaru SCEXAO system with modulation (left) and without modulation (right). 


\section{A NEAR-INFRARED WAVEFRONT SENSOR FOR KECK AO}

In this section we describe the conceptual design for a near-IR wavefront sensor for the Keck II AO system. The wavefront sensor is being designed to support both NGS and LGS AO. In LGS AO it would be a low order sensor, at minimum providing high bandwidth tip-tilt sensing, with the intention to also provide low bandwidth focus using the LIFT algorithm. In NGS AO it would be the high order sensor.

A dichroic beamsplitter just before the NIRC2 science camera would reflect the light to the near-IR wavefront sensor and transmit the light to NIRC2.

\subsection{Pyramid Wavefront Sensor Conceptual Layout}

A conceptual view of the planned Keck PWFS layout is shown in Figure 11. Lenses L1 and L2 will be used to convert the $\mathrm{f} / 15 \mathrm{AO}$ output to the desired focal ratio on the pyramid. The relay produces an intermediate pupil image, located a distance $f_{1}$ after lens $L_{1}$, where a mirror will be placed to modulate the beam on the tip of the pyramid to increase the dynamic range of the sensor. The pyramidal prism is located in a focal plane and divides the incoming beam into four beams each of which is then imaged on the detector at a pupil plane. Each pupil image will be $\sim 20$ pixels in diameter with each pixel corresponding to the $7 \mathrm{~mm}$ physical deformable mirror actuator spacing, which in turn corresponds to 56 $\mathrm{cm}$ as projected on the telescope primary mirror.

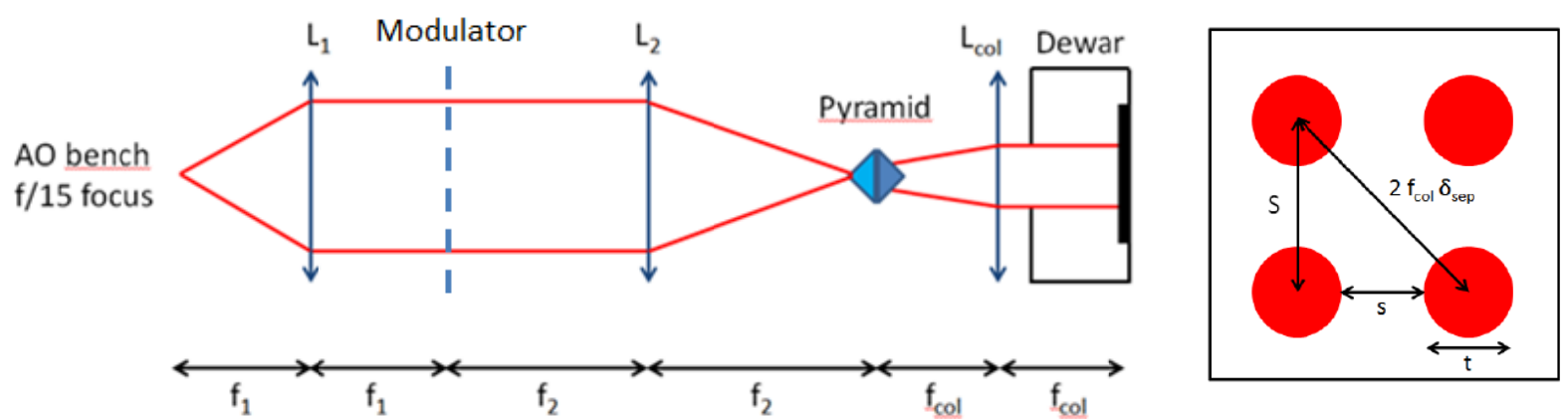

Figure 11: Left: Conceptual layout of the PWFS optics. Right: The four pupil images on the detector.

According to the Subaru visible PWFS experience, modulation in a circular path, with a radius of approximately three times the diffraction-limited image diameter $(0.1$ " for Keck at H-band), will be required for the PWFS to work well with faint guide stars. From the Large Binocular Telescope (LBT) visible PWFS experience the range is two to six times the diffraction-limited image diameter [18]. A Physique Instrumente 330.30 tip-tilt stage will be used for this purpose. Modulation is achieved by using two sine waves for $\mathrm{x}$ and $\mathrm{y}$ modulation. Synchronization will be achieved with a comparator monitoring one of the sine waves to trigger the camera readout at a particular voltage.

It will be important to accurately position and maintain the science target since the L-band vortex coronagraph mask will be a science mode that uses the PWFS. The modulator mirror will therefore also be used for small pointing corrections to keep the star centered on the pyramid and to provide differential atmospheric refraction correction between the science and PWFS wavelengths during an exposure. Offsets of the star from the tip of the pyramid by up to one diffractionlimited diameter should result in no loss of performance and are sufficient to address differential atmospheric refraction. An $\mathrm{x}$ and $\mathrm{y}$ dc offset added to the two modulating sine waves will be used to offset the star from the tip of the pyramid.

Pupil stability is critical and should be held to a fraction of a pixel on the camera to prevent instability in the higher order modes. The AO rotator can be used to maintain a fixed pupil orientation which will help with pupil stability. The Keck PWFS design includes capability to control the collimating lens centering $\left(\mathrm{L}_{\mathrm{col}}\right.$ in Figure 11$)$ to maintain the pupil position at $1 \mathrm{~Hz}$ rates, sufficient to compensate for thermal variations and the small nutations of the telescope pupil.

\subsection{Combined Low Order and Pyramid Wavefront Sensor}

A conceptual layout for a combined NIR tip-tilt and PWFS is shown in Figure 12. The tip-tilt sensor optical design is driven by the pupil size on the modulator and the required plate scale on the detector. For a $5 \mathrm{~mm}$ pupil size then $\mathrm{f}_{1}=75$ $\mathrm{mm}$ for L1. If the SAPHIRA detector were placed directly at the AO focus the plate scale would be $33 \mathrm{mas} / \mathrm{pixel}$. Given the results from Figure 5 a 1-to-1 relay would work well for the tip-tilt sensing $\left(f_{2 t t}=f_{1}\right)$ and the overall length of the tiptilt relay, from $\mathrm{AO}$ focus to detector, would be $4 \mathrm{f}_{1}=300 \mathrm{~mm}$. 


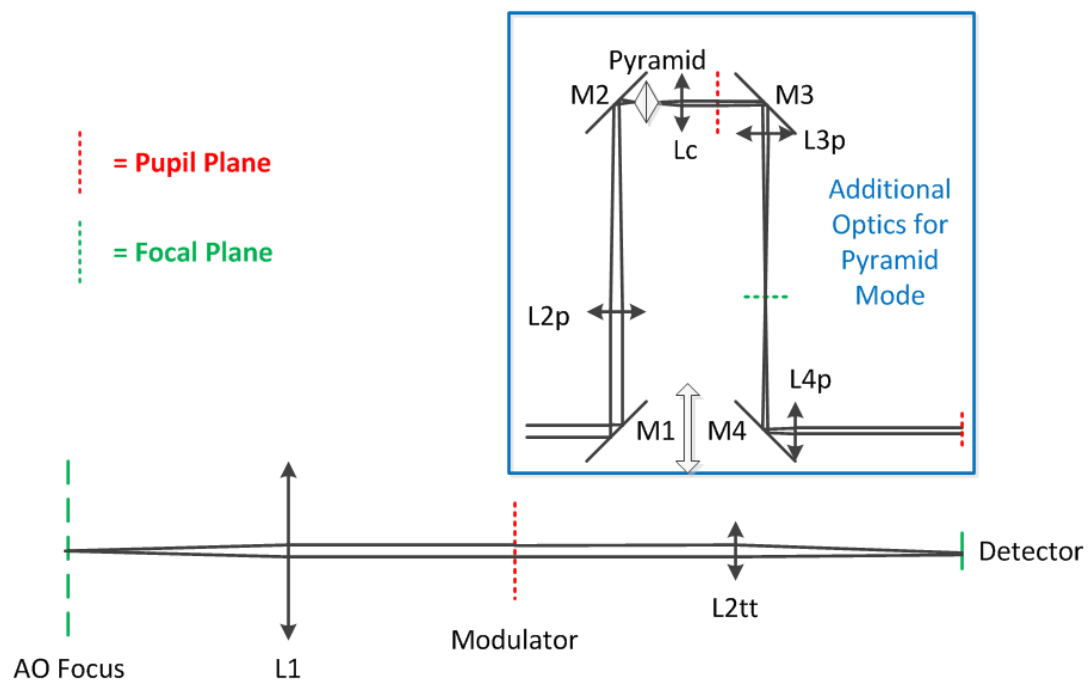

Figure 12: Conceptual optical layout for a combined near-IR tip-tilt sensor and PWFS. The bottom image is the tip-tilt sensor. A lens L1 collimates the light and reimages the telescope pupil on a modulator (a tip-tilt mirror). A second lens L2tt focuses the light onto the detector. The additional optics for the PWFS mode are shown in the blue rectangle. The $L 2 p$ through $L 3 p$ components could be fixed and a stage could insert M1, M4 and L4p into the beam while removing $L 2 t t$. L2p focuses the light onto the tip of a pyramid while Le collimates the light and produces a pupil image. $\mathrm{L} 3 \mathrm{p}$ and $\mathrm{L} 4 \mathrm{p}$ relay the pupil image onto the detector.

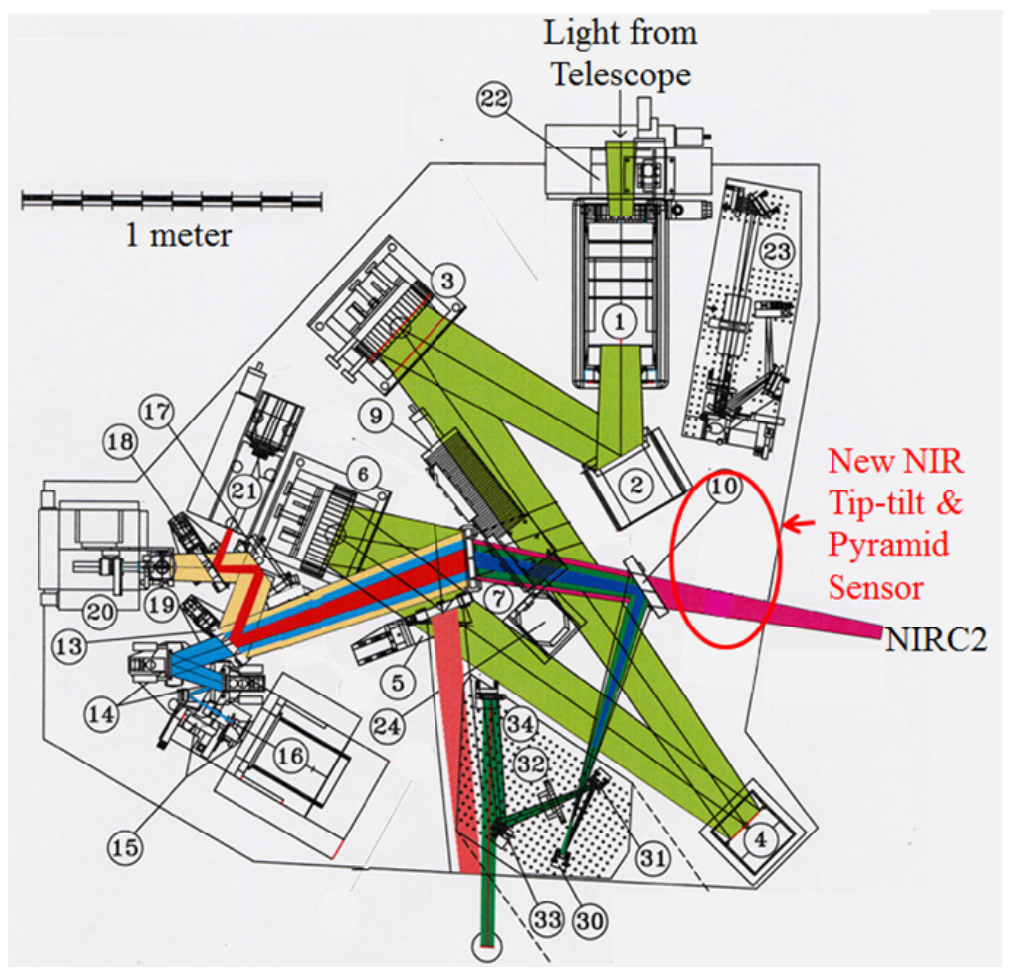

Figure 13: Keck II AO bench layout showing the location of the new combined near-IR tip-tilt sensor and PWFS. The sensor would be fed by a dichroic beamsplitter located at the edge of the AO bench just before the NIRC2 science instrument. The NIRC2 science path includes an imager rotator (item 1), a fast tip-tilt mirror (2), an offaxis parabola (3), the deformable mirror (4), a second off-axis parabola (6) and an IR transmissive dichroic (7).

The $\mathrm{L} 2 \mathrm{p}$ focal length is chosen for the required $\mathrm{f} / 36$ on the tip of the prism $\left(\mathrm{f}_{2 \mathrm{p}}=180 \mathrm{~mm}\right)$. The collimation lens, Lc, focal length is chosen for the required pupil size on the detector; for the case of one subaperture $(0.5625 \mathrm{~m})$ per pixel this 
requires a $10 \mathrm{~m} / 0.5625=17.78$ pixel pupil $\left(f_{c}=15.36 \mathrm{~mm}\right)$. L3p and $\mathrm{L} 4 \mathrm{p}$ were chosen to provide a unit magnification projection of the pupil onto the detector with a back focal distance of $60 \mathrm{~mm}$ since the detector is located $\sim 40 \mathrm{~mm}$ behind the camera window.

The positions of mirrors M1 to M4 were chosen to provide a reasonable separation from the modulator and detector while providing the necessary extra path length. In the selected layout M1 is $30 \mathrm{~mm}$ after the modulator and M4 is 80 $\mathrm{mm}$ from the detector. The M1 to M4 (and M2 to M3) distance is therefore $40 \mathrm{~mm}$, and the M1 to M2 distance is 240.4 $\mathrm{mm}$. For this case the pyramid is $49.7 \mathrm{~mm}$ after M3 (as opposed to what is shown in Figure 12).

The required location for the combined near-IR tip-tilt sensor and PWFS on the existing Keck II AO bench is shown in Figure 13. A dichroic beamsplitter located just in front of the NIRC2 science instrument would reflect the field to the combined near-IR sensor. An initial Zemax optical design of the tip-tilt sensor path is shown in Figure 14. After reflection by the dichroic, a fold mirror, large enough to cover the full field, folds the beam down toward the AO bench. This is followed by another dichroic, just large enough for the NIR WFS field, that folds the beam parallel to the AO bench and toward the NIR WFS (the transmitted light will go to a fiber injection unit). Everything from the NIR WFS field dichroic to the Selex detector would be on a xy-translation stage to access the full 120 arcsec diameter field. The light transmitted by the near-IR WFS field dichroic will go to a single mode fiber injection unit (PI: D. Mawet) that will feed the NIRSPEC spectrograph.

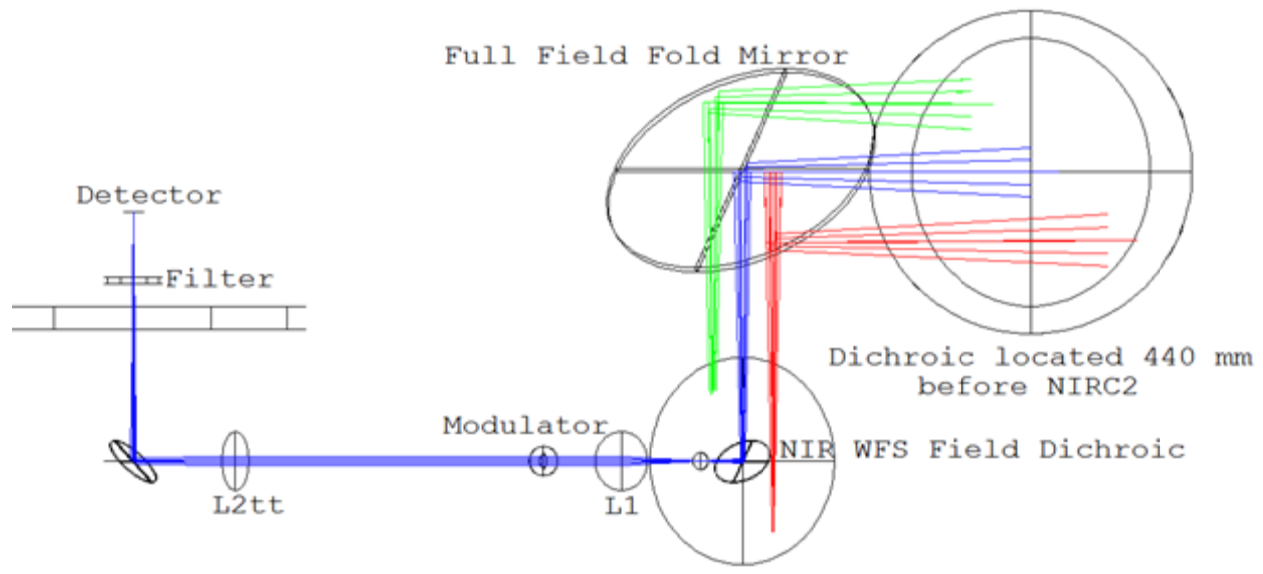

Figure 14: Preliminary Zemax optical design for the NIR tip-tilt sensor showing the optical path from the dichroic just before NIRC2 to the Selex detector.

\subsection{Camera}
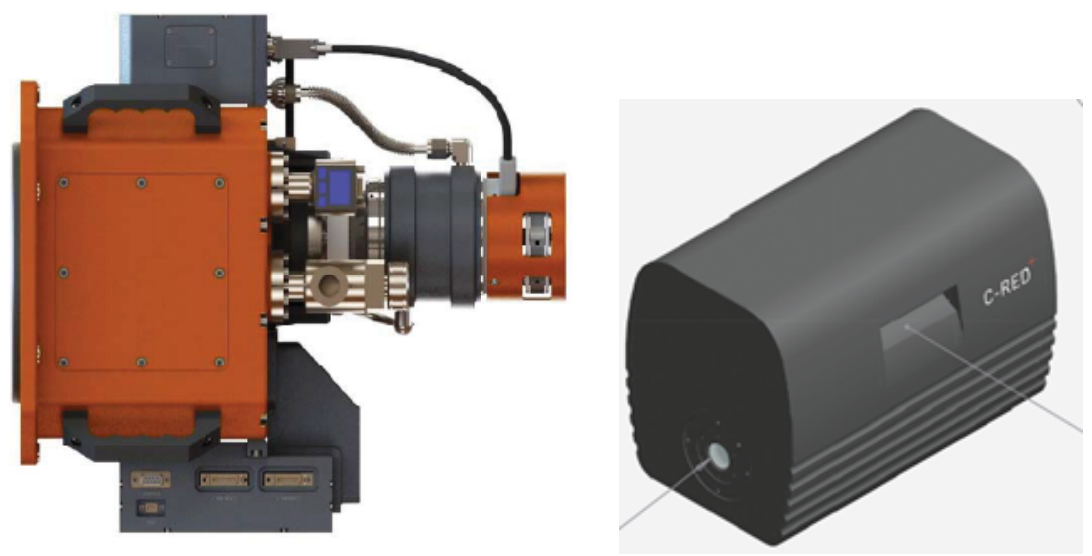

Figure 15: Left: University of Hawaii camera dewar (orange) from GL Scientific. The detector is located $50.5 \mathrm{~mm}$ from the front mounting surface at left. The overall package length is $457 \mathrm{~mm}$ including the cryocooler extending to the right. Right: First Light Inc.'s C-Red One camera head is $341 \mathrm{~mm}$ long x $250 \mathrm{~mm}$ high x $215 \mathrm{~mm}$ wide; the entrance window is $65 \mathrm{~mm}$ from the bottom of the mounting surface. 
Figure 15 shows two Selex SAPHIRA-based camera systems, developed by the University of Hawaii (UH) and First Light Imaging (FLI) [19], respectively. The UH camera uses a GL Scientific dewar cooled by an actively vibration damped Sunpower CryoTel-GT Stirling cooler, coupled to a Lakeshore temperature controller and a chiller. The First Light Inc. C-RED one camera is cooled to $80 \mathrm{~K}$ using a high reliability, integrated pulse tube. Both camera options would fit on the AO bench in the space allowed by the Figure 14 optical design.

The UH camera saw first light at the NASA IRTF in May, 2014 and has subsequently been deployed to both the Palomar $1.5 \mathrm{~m}$ with Robo-AO and to SCExAO on Subaru (where it is currently in use). UH has procured two additional camera systems, one of which is intended for observations with Keck. The UH cameras are operated using a UH "Pizza Box" controller [20] which currently operates with $1 \mathrm{MHz}$ pixel rate, corresponding to a full frame rate of $400 \mathrm{~Hz}$ with $2 \mathrm{kHz}$ rate for a $128 \times 128$ sub-array and $8 \mathrm{kHz}$ for a 64 x64 sub-array. The controller is capable of $2 \mathrm{MHz}$ pixel rate which would double these frequencies.

\subsection{Real-time Controller}

The PWFS will be used to control the Keck AO system's tip-tilt and deformable mirrors. The processing of the PWFS pixel data through the PWFS algorithm could occur in the existing Keck AO real-time controller (RTC) or in a separate fast processor. In either case the Keck RTC [21] would still provide the driving control of the mirrors.

We intend to develop a separate flexible RTC to accept the PWFS pixels and to process them through the PWFS algorithm. This approach has the additional benefit of allowing test algorithms to be easily programmed. The new RTC will be based on the visible PWFS controller developed at Subaru. This RTC is very simple, a Dell server and the Ubuntu Linux open source software platform, with no custom hardware. Computationally intensive operations are moved to GPUs. The code was specifically

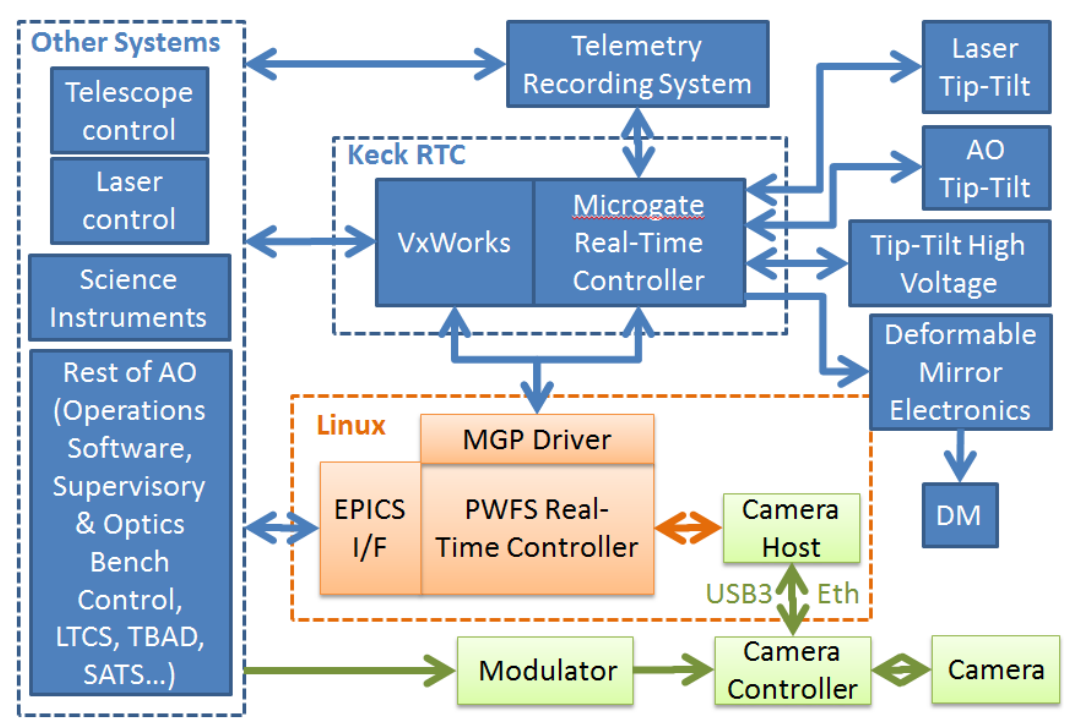

Figure 16: The new PWFS real-time controller (orange) and camera system (green) will interface to the existing Keck systems (blue). The camera and modulator are part of the PWFS system on the AO bench; the modulator drives both the modulation mirror and the PWFS camera readout. The integrated camera host computer and PWFS real-time controller will be located in the AO electronics room next to the Microgate real-time controller.

designed to support the new generation of high speed diffraction-limited wavefront sensors (e.g. for the PWFS diffraction outside of the geometric pupils is taken into account, and the software includes high speed zero-point offsetting capability). A simplified version of the Subaru system with reduced computational requirements will be implemented for our proposed project.

The new PWFS RTC will interface to the existing Keck AO system via two interface layers as shown in Figure 16. A simplified version of the VxWorks Microgate Protocol (MGP; C code) driver will be ported to the Linux machine to write data packets to the Microgate RTC. The MGP driver will send the deformable and tip-tilt mirror commands to the Microgate RTC at up to $2 \mathrm{kHz}$ rates over an existing private gigabit Ethernet network. An EPICS layer will be added to the PWFS RTC to support communication with the rest of the observatory over the observatory network.

This interface approach minimizes the need to modify or replace existing hardware and software interfaces, and hence the impact on the operational system. It also makes use of the existing AO tools. For example: (1) tip-tilt and focus offloading loops to the telescope will continue to be handled by the existing Keck systems monitoring the tip-tilt and deformable mirrors; (2) deformable mirror offsets for image sharpening on the vortex coronagraph and speckle nulling 
will continue to be determined by the operational system and will be passed as a calibration file to the PWFS RTC; and (3) pointing offsets to register the science target on the vortex coronagraph and compensate for differential atmospheric refraction will continue to be determined by the operational system and will be passed to the modulator driver.

\section{CONCLUSIONS}

Wavefront sensing with the low noise near-IR detectors that have recently become available offers considerable sky coverage improvements for both LGS and NGS AO. In LGS mode these sensor can be used for tip-tilt as well as for measuring the low order aberrations introduced by the LGS. For high order NGS AO the low noise and flexible readout of the Selex SAPHIRA e-APD arrays is particularly optimal and supports improved AO correction.

A combined near-IR tip-tilt and pyramid wavefront sensor design is described which would support Keck II LGS and NGS AO with the NIRC2 and NIRSPEC science instruments. The tip-tilt sensor mode is being designed to support the determination of low order modes with the LIFT algorithm that has shown promising laboratory results.

\section{ACKNOWLEDGEMENTS}

The W. M. Keck Observatory is operated as a scientific partnership among the California Institute of Technology, the University of California, and the National Aeronautics and Space Administration. The Observatory was made possible by the generous financial support of the W. M. Keck Foundation. The Keck I near-IR tip-tilt sensor was supported by the National Science Foundation under Grant No. AST-1007058 and by the Gordon and Betty Moore Foundation under Grant No. 4046.

The authors wish to recognize and acknowledge the very significant cultural role and reverence that the summit of Maunakea has always had within the indigenous Hawaiian community. We are most fortunate to have the opportunity to conduct observations from this mountain.

\section{REFERENCES}

[1] Colavita, M., Wizinowich, P., Akeson, R., Ragland, S., Woillez, J., Millan-Gabet, R., Serabyn, E., et al., "The Keck Interferometer," PASP 125, 1226-1264 (2013).

[2] Gendron, E., Lacombe, F., Rouan, D., Charton, J., Collin, C., Lefort, B., Marlot, C., Michet, G., Nicol, G., Pau, S., Phan, V., Talureau, B., Lizon, J.-L., Hubin, N., "NAOS infrared wavefront sensor design and performance," Proc. SPIE 4839, 195 (2003).

[3] Peter, D., Feldt, M., Henning, T., Hippler, S., Aceituno, J., Montoya, L., Costa, J., Dorner, B., "PYRAMIR: Exploring the On-Sky Performance of the World's First Near-Infrared Pyramid Wavefront Sensor," PASP 122, 63 (2010).

[4] Femenia Castella, B., Wizinowich, P., Rampy, R., Cetre, S., Lilley, S., Lyke, J., Ragland, S., Stomski, P., van Dam, M., "Status and new developments with the Keck I near-infrared tip-tilt sensor," Proc. SPIE 9909-79 (2016).

[5] Feautrier, P., Gach, J.-L., Wizinowich, P., "State of the art IR cameras for wavefront sensing using e-APD MCT arrays," Adaptive Optics for Extremely Large Telescopes Conference 4 (Los Angeles, 2015).

[6] Guieu, S., Feautrier, P., Zins, G., Le Bouquin, J. -B., Stadler, E., Kern, P., Rothman, J., Tauvy, M., Coussement, J., de Borniol, E., Gach, J. -L., Jacquard, M., Moulin, T., Rochat, S., Delboulb, A., Derelle, S., Robert, C., Vuillermet, M., Mérand, A., Bourget, P., "RAPID, a revolutionary fast optical to NIR camera applied to interferometry," Proc. SPIE 9146, 91461N (2014).

[7] Atkinson, D., Hall, D., Baranec, C., Baker, I., Jacobson, S., Riddle, R., "Observatory Deployment and Characterization of SAPHIRA HgCdTe APD Arrays," Proc. SPIE 9154, 914519 (2014).

[8] Baranec, C., Atkinson, D., Riddle, R., Hall, D., Jacobson, S., Law, N., Chun, M., "High-speed Imaging and Wavefront Sensing with an Infrared Avalanche Photodiode Array," ApJ 809, 70 (2015).

[9] Finger, G., Baker, I., Alvarez, D., Ives, D., Mehrgan, L., Meyer, M., Stemeier, J., Weller, H., "SAPHIRA detector for infrared wavefront sensing," Proc. SPIE 9148, 914817 (2014).

[10] Atkinson, D. et al., "Next-generation performance of SAPHIRA HgCdTe APDs," Proc. SPIE 9915-22 (2016). 
[11] Hall, D. et al., "Towards the next generation of L-APD MOVPE HgCdTe arrays: beyond the SAPHIRA 320x256," Proc. SPIE 9915-21 (2016).

[12] Rampy, R., Femenia, B., Lyke, J., Wizinowich, P., Cetre, S., Ragland, S., Stomski, P., "Near-infrared tip-tilt sensing at Keck: System architecture and on-sky performance," Adaptive Optics for Extremely Large Telescopes Conference 4 (Los Angeles, 2015).

[13] Wizinowich, P., Le Mignant, D., Bouchez, A., Campbell, R., Chin, J., Contos, A., van Dam, M., Hartman, S., Johansson, E., Lafon, R., Lewis, H., Stomski, P., Summers, D., Brown, C., Danforth, P., Max, Pennington, D., "The W. M. Keck Observatory Laser Guide Star Adaptive Optics System: Overview," PASP 118, 310 (2006).

[14] Meimon, S., Fusco, T., Mugnier, L., "LIFT: a focal-plane wavefront sensor for real-time low-order sensing on faint sources," Optics Letters 35, 3036 (2010).

[15] Plantet, C., Wizinowich, P., Fusco, T., Neichel, B, Ragland, S., Rampy, R., "Lift on Keck: analysis of performance and first experimental results," Proc. SPIE 9909-233 (2016).

[16] Femenia Castella, B. et al., "Commissioning and first light results of an L'-band coronagraph with the Keck II adaptive optics NIRC2 science instrument," Proc. SPIE 9909-76 (2016b).

[17] Plantet. C., Meimon, S., Conan, J.-M., Fusco, T., "Revisiting the comparison between the Shack-Hartmann and the pyramid wavefront sensors via the Fisher information matrix," Optics Express 23, 28619 (2015).

[18] Esposito, S., Riccardi, A., Pinna, E., Puglisi, A., Quiros-Pacheco, F., Arcidiacono, C., Xompero, M., Briguglio, R., Agapito, G., Busoni, L., Fini, L., Argomedo, J., Gherardi, A., Brusa, G., Miller, D., Guerra, J., Stefanini, P., Salinari, P., "Large Binocular Telescope Adaptive Optics System: New achievements and perspectives in adaptive optics," Proc. SPIE 8149, 814902 (2011).

[19] Gach, J.-L., Feautrier, P., Stadler, E., Greffe, T., Clop, F., Lemarchand, S., Carmignani, T., Boutolleau, D., Baker, I., "C-RED one: ultra-high speed wavefront sensing in the infrared made possible," Proc. SPIE 9909-41 (2016).

[20] Lockhart, C. et al., "The PizzaBox-1 array controller: a high speed low noise array controller for Teledyne H2RG, H4RG, and Selex SAPHIRA devices," Proc. SPIE 9915-104 (2016).

[21] Johansson, E., van Dam, M., Stomski, P., Bell, J., Chin, J., Sumner, R., Wizinowich, P., Biasi, R., Andrighettoni, M., \& Pescoller, D., "Upgrading the Keck AO wavefront controllers," Proc. SPIE 7015, 7015-121 (2008). 